\title{
Characterization of the Psoriasis-like Inflammation in the Imiquimod Rat Model using Dermal Open Flow Microperfusion
}

T. Birngruber ${ }^{1}$, A. Eberl ${ }^{1}$, D. Kollmann ${ }^{1}$, M. Bodenlenz ${ }^{1}$, P. Florian ${ }^{3}$, A.Subramaniam ${ }^{3}$, S. Kainz ${ }^{1}$, G. Rauter ${ }^{1,4}$, F. Sinner ${ }^{1,2}$

CONTACT

JOANNEUM RESEARCH

Forschungsgesellschaft mbH

HEALTH

Institute for Biomedicine and Health Sciences

Neue Stiftingtalstrasse 2

8010 Graz

Phone +43 $316876-4000$ Fax +43 316876 9-4010

health@joanneum.at

www.joanneum.at/health

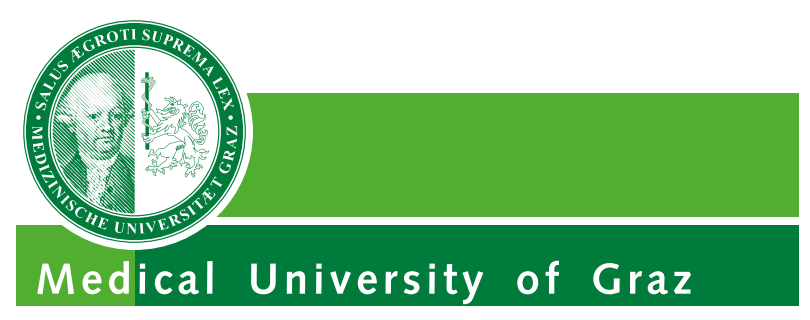

2 Medical University of Graz

Division of Endocrinology and Diabetology

Graz, Austria

\section{SANOFI GENZYME פ}

${ }^{3}$ Sanofi-Genzyme

Framingham, MA, USA and Sanofi Frankfurt, Germany

${ }^{4}$ Division of Biomedical Research Medical University of Graz Graz, Austria

References

C. Dragatin et al. "Secukinumab distributes into dermal interstitial fluid of psoriasis patients as demonstrated Exp. Dermatol., 2016.

F. Kolbinger et al. "B-defensin-2 is a responsive biomarker of IL-17A-driven

skin pathology in psoriasis,
Allergy Clin Immunol, 2016 ,

Modenlenz et [3 ] "Open Flow Microperfusion as Dermal Pharmacokinetic Approach to Evaluate Topical Broequivalence",

van der Fits et al. "Imiquimodduced psoriasis-like skin inflammation in mice is mediated via the IL-23/L-17 axis", J Immunol., 2009,

B.Y. Kim et al. "Histopathological findings are associated with the clinical types of psoriasis but not with the corresponding lesional psoriasis severity index." Ann Dermatol. 2015
Purpose

Topically applied imiquimod has been shown to induce psoriasis-like skin inflammation in mice. The use of larger animals such as rats would enable the use of clinical research techniques such as dermal Open-Flow Microperfusion (dOFM). This minimally-invasive clinical method has proven to enable continuous sampling of different drugs and cytokines in inflamed and healthy skin in humans in vivo in PK-PD as well as topical bioequivalence trials ${ }^{[1-3]}$. We tested whether psoriasis-like inflammation can be induced by using imiquimod in a rat model and characterized any effects of imiquimod and a known immunosuppressant by using clinical skin scores and cytokine profiles from dOFM sampling of inflamed and healthy skin.

\section{Methods}

- Sprague-Dawley rats, $\mathrm{n}=24$

- Day 1-8, topical treatment, $\mathrm{n}=24$

IMQ-treated (IMQ+), right back: Once daily topical imiquimod (IMQ) cream to introduce psoriasis-like skin inflammation

- Untreated (IMQ-), left back: № topical treatment, i.e. non-lesional skin

- Day 1-8, oral treatment, 2 groups each $n=12$ :

- Dex: Once daily oral dexamethasone to inhibit inflammation $(1-2 \mathrm{mg} / \mathrm{kg})$

- Vehicle: Vehicle (placebo) or no oral treatment as control group

- Day 9, dermal interstitial fluid sampling by dOFM

- 3 dOFM probes for sampling from IMQ+ skin

3 dOFM probes for sampling from untreated skin

$\rightarrow$ Fig. 1 shows the dOFM protocol

- Fig. 2 shows the dOFM principle

- Skin evaluation: erythema, skinfold thickness, scaling, histopathology scores (HPS) ${ }^{[5]}$

- Dermal interstitial fluid (dOFM): Forty cytokines using rodent panels (MSD rodent panel, Ampersand rodent panel)

Results \& Discussion The daily topical IMQ dose induced visible skin inflammation in all rats within 4 days (Fig. 3) which is similar to mice ${ }^{[4]}$. After 8 days, the lesions were fully developed. Day 9 data demonstrated ( Fig. 4, Table 1):

- that IMQ had induced psoriasis-like skin inflammation: Increased erythema, skinfold-thickness, histopathology scores, and significantly increased release of a number of cytokines in treated vs. untreated skin. IL-17A was only quantifiable in IMQ-treated skin. Still, IL17A and IL17F levels were very low on day 9, however, these low levels are in agreement with the reduced expression of IL-23, IL-17A, and IL-17F in the IMQ model in mice after 8 days ${ }^{[4]}$ as IMQ only transiently induces cytokines of the $\mathrm{LL}-23 / \mathrm{L}-17$ axis in skin.

- the efficacy of dexamethasone to inhibit the inflammatory effect of topical IMQ.

- an effect of IMQ and dexamethasone on the dermal immune cell population - see poster \# 488

Conclusions

- Topical imiquimod rapidly induces psoriasis-like skin inflammation in rats.

- Oral dexamethasone inhibits the inflammatory effect in rats.

- This imiquimod rat model can be used to screen novel psoriasis drugs.

- dOFM enabled cytokine and immune cell sampling from rat skin in-vivo. This study demonstrates that this clinical tool is also highly informative in preclinical research.
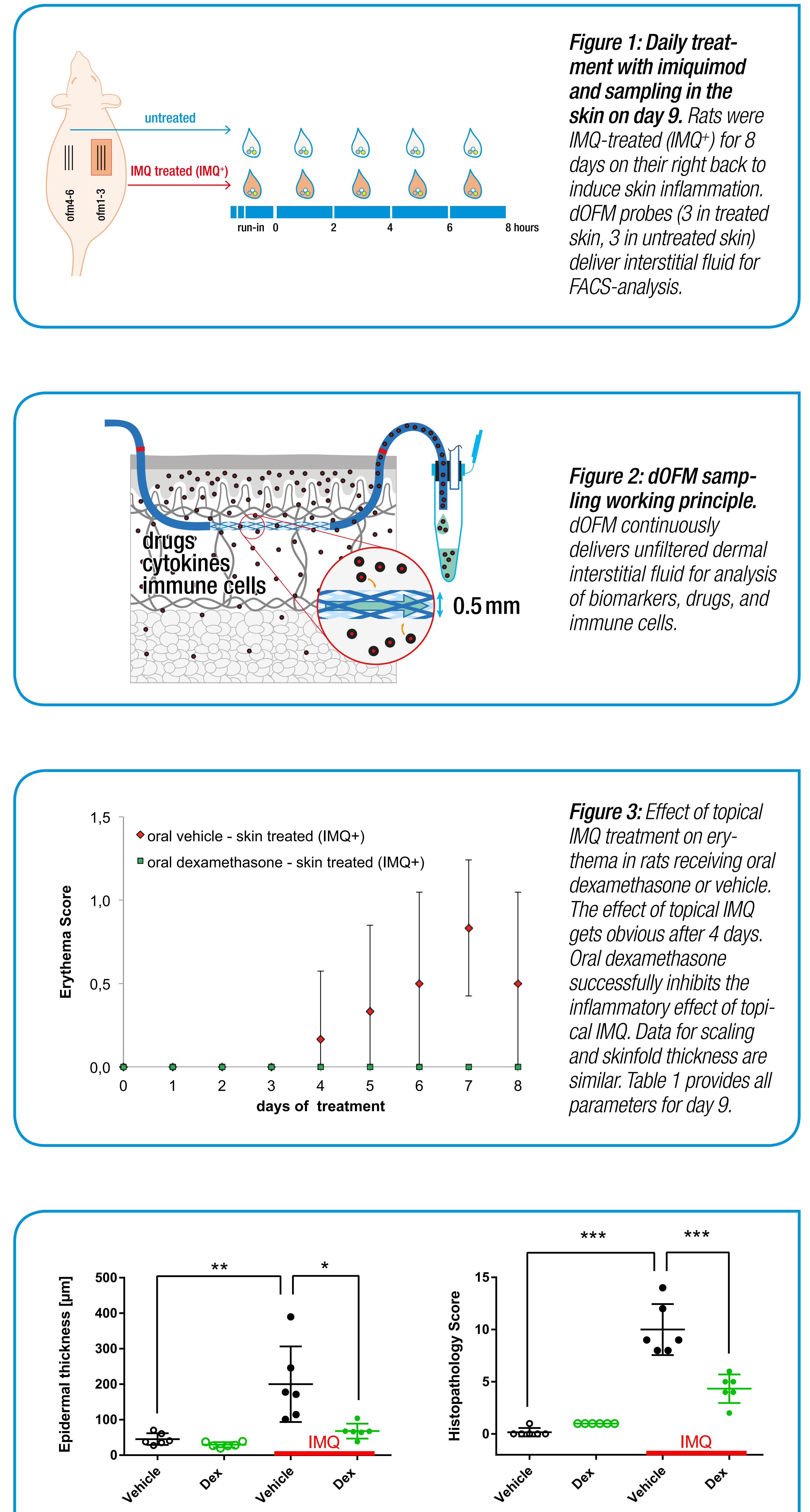

Figure 4: Epidermal thickness (left panel) and histopathology scores (HPS, right panel) from skin biopsies on day 9. The HPS score was adapted from Kim et. al. 2015. ${ }^{[5]}$

Table 1: Effect of treatments on skin appearance on day 9

\begin{tabular}{|c|c|c|}
\hline & \begin{tabular}{|c|} 
IMQ effect \\
(IMOQ vs. \\
untreated skin)
\end{tabular} & $\begin{array}{l}\text { Dex efficacy } \\
\text { (Oral Dex vs. } \\
\text { vehicle) }\end{array}$ \\
\hline & $\mathrm{N}=12$ rats & $\mathrm{N}=12$ rats \\
\hline Skinfold thickness & $P<0.05$ & $P<0.05$ \\
\hline Erythema & $P<0.05$ & $P<0.05$ \\
\hline Scaling & $P<0.05$ & $P<0.05$ \\
\hline Epidermal thickness' & $P<0.05$ & $P<0.05$ \\
\hline HPS $^{1}$ & $P<0.05$ & $P<0.05$ \\
\hline
\end{tabular}

Table 2: Effects of treatments on intradermal cytokines/markers on day 9

\begin{tabular}{|c|c|c|}
\hline & $\begin{array}{l}\text { IMQ effect } \\
\text { (IMQ+oss. } \\
\text { untreated skin) }\end{array}$ & $\begin{array}{l}\text { Dex efficacy } \\
\text { (Oral Dex ves. } \\
\text { vehicle) }\end{array}$ \\
\hline & $\mathrm{N}=12$ rats & $\mathrm{N}=12$ rats \\
\hline IL-10 & $P<0.05$ & $P<0.05$ \\
\hline IL-12p70 & $P<0.05$ & $P<0.05$ \\
\hline IFN $\beta$ & $P<0.05$ & $P<0.05$ \\
\hline IL-1a & ns & $\mathrm{P}<0.05$ \\
\hline IL-6 & ns & $P<0.05$ \\
\hline IL-17A & $n s^{2}$ & $\mathrm{p}<0.10$ \\
\hline IL-17F & ns & ns \\
\hline
\end{tabular}

